Relations industrielles

Industrial Relations

\title{
Changes in the Location of Manufacturing in the United States, Victor R. Fuchs, New Haven and London. Yale University Press, 1962. 566 pages.
}

\section{Bertrand Belzile}

Volume 18, numéro 2, avril 1963

URI : https://id.erudit.org/iderudit/1021440ar

DOI : https://doi.org/10.7202/1021440ar

Aller au sommaire du numéro

Éditeur(s)

Département des relations industrielles de l’Université Laval

ISSN

0034-379X (imprimé)

1703-8138 (numérique)

Découvrir la revue

Citer ce compte rendu

Belzile, B. (1963). Compte rendu de [Changes in the Location of Manufacturing in the United States, Victor R. Fuchs, New Haven and London. Yale University Press, 1962.566 pages.] Relations industrielles / Industrial Relations, 18(2), 282-283. https://doi.org/10.7202/1021440ar

Tous droits réservés (C Département des relations industrielles de l’Université Laval, 1963
Ce document est protégé par la loi sur le droit d'auteur. L'utilisation des services d’Érudit (y compris la reproduction) est assujettie à sa politique d'utilisation que vous pouvez consulter en ligne.

https://apropos.erudit.org/fr/usagers/politique-dutilisation/ 
Les négociations collectives débutent et se poursuivent selon le processus normal : négociations, négociations directes, conciliation, conseil de conciliation, et grève. Sous cet optique, les parties négocient mais ne perdent pas de vue cette réalité inéluctable qu'elles évoluent dans le cadre d'un service public aux malades et hospitalisés.

Le droit de grève, inaliénable et inprescriptible, est reconnu par la législation, mais l'exercice de ce droit et ses modalités d'application sont discutés et négociés entre les trois parties. S'il n'y a pas accord, sur telles modalités, chaque partie prend ses responsabilités et, en définitive, il $y$ a appel au tribunal de l'opinion publique.

Il reste au législateur l'ultime recours, à la mesure d'exception, décrété pour état d'urgence.

En définitive, il doit y avoir $\ll$ un particularisme du droit du travail $>$ spécialement dans le secteur hospitalier.

\section{RECENSIONS - BOOK REVIEWS}

Changes in the Location of Manufacturing in the United States, Victor $\mathbf{R}$. Fuchs, New Haven and London. Yale University Press, 1962. 566 pages.

Cette monographie est une étude des changements dans la localisation des manufactures aux Etats-Unis et une analyse de la mobilité des industries et des différences territoriales dans les taux de croissance. L'excellente qualité de cet ouvrage repose sur l'utilisation scientifique d'une abondante documentation statistique originale d'une part et sur la tentative systématique de vérifier de nombreuses hypothèses ayant cours en localisation industrielle d'autre part.

A partir de statistiques détaillées du recensement des manufactures, l'auteur étudie la redistribution des activités industrielles par région. Le nombre des industries étudiées dépasse deux cents
(200) et celui des groupes industriels atteint vingt (20). Les régions analysées sont les états et des groupes d'états.

En plus de constater les changements survenus dans la localisation des industries, M. Fuchs essaie de les expliquer. Selon lui, les différentiels de croissance peuvent provenir des différences territoriales dans la structure industrielle ou dans la croissance des industries spécifiques. Il reconnaît que c'est le dernier type de différences qui influence davantage la redistribution des manufactures.

L'auteur étudie précisément l'influence sur la localisation industrielle de nombreuses variables, dont les suivantes : la demande du consommateur, les matières premières, les taxes, le commerce extérieur, les niveaux de salaire, le degré de syndicalisation, l'espace, le climat, etc. Il tente également de vérifiè la signification à cet égard de d'autres 
caractéristiques des industries telles que le taux de croissance, les gages par rapport à la valeur ajoutée, la concentration de la propriété, etc. Une attention toute spéciale est accordée aux deux types dindustries, market oriented et labor-oriented s.

Une autre partie du volume décrit la croissance comparative des régions en tant que telles et essaie d'en expliquer les différences. Puis les groupes industriels sont identifiés selon leur degré de mobilité interrégionale et les facteurs prédominants sont recherchés.

L'auteur a reconnu une importance prépondérante sur la localisation des manufactures aux facteurs suivants : l'attraction des ressources naturelles et des matières premières $(1 / 3)$, la maind'oeuvre abondante, à bon marché et non-organisée $(1 / 3)$ et enfin les changements dans la demande $(1 / 3)$.

Enfin l'auteur signale que les changements de localisation survenus entre 1929 et 1954 ont eu trois résultats importants : a) la plupart des industries sont maintenant plus dispersées géographiquement; b) la plupart des régions sont moins dépendantes d'un seul groupe industriel pour l'emploi manufacturier ; c) les différences interrégionales dans la structure industrielle ont été réduites de façon substantielle.

\section{Bertrand Belzine}

Economics in a Canadian Setting, par Mark Keith Inman. The Copp Clark Publishing Co. Ltd., 1959, 771 pages.

\& Economics in a Canadian Setting * est avant tout un manuel d'introduction à l'économique. Il suffit d'énumérer quelques titres de chapitres pour s'en rendre compte : la comptabilité du revenu national, les fluctuations économiques, les finances publiques, la consommation, les politiques de prix et de volume dans différentes conditions de production, le commerce international, etc.

Ce qui distingue ce volume des autres que nous connaissons sur le sujet, c'est que l'auteur s'efforce d'illustrer les exposés théoriques par des exemples tirés de l'économie canadienne. Bien plus, dans la première partie de son volume, M. Inman décrit assez longuement les institutions économiques cana- diennes dans les domaines des affaires, de la finance et de l'organisation du travail. La lecture de ce livre fournira aux non-initiés une connaissance générale mais systématique de l'économie canadienne et les incitera à approfondir les principes qui en dictent le fonctionnement.

Les non-initiés se sentiront ainsi moins dépaysés dans leurs démarches vers une connaissance plus grande de la science économique. Mais il existe un intérêt additionnel pour eux : l'auteur, en plus de référer souvent à l'économie canadienne, pose plusieurs questions qui correspondent bien aux difficultés premières de compréhension de l'économique. Au lieu de faire de longs développements théoriques, M. Inman énonce l'essentiel de chaque théorie ou phénomène économique et l'illustre par un exemple simple et approprié pour en faciliter la compréhension.

De plus, la présentation elle-même du texte aide beaucoup le lecteur à se situer continuellement dans le sujet traité, à l'intérieur du contexte général. En effet, le texte est abondamment subdivisé et un titre coiffe à peu près chaque paragraphe. Les nombreux tableaux et graphiques facilitent également la compréhension de l'exposé.

Bref, Economics in a Canadian Setting ne présente à vrai dire aucune originalité dans son contenu, mais constitue pour un Canadien une excellente introduction à l'économique pour ne pas dire à l'économie canadienne comme telle. Ce qui caractérise avant tout ce volume, c'est sa haute valeur pédagogique.

\section{Bertrand Belzile.}

Sécurité sociale et conflits de classes. Centre d'études des Relations sociales. Préface de Pierre Laroque. Collection < Relations sociales », Economie et Humanisme - Les Editions ouvrières, Paris, 1962, 167 pages.

La sécurité sociale est un facteur essentiel de transformation sociale. Elle doit son origine au souci de faire disparaître ou du moins d'atténuer l'insécurité dans laquelle vivent une grande partie des individus et des familles. Or, c'est précisément cette inégalité dans la possibilité de faire face aux risques sociaux qui est une des causes les plus 